\title{
Critical Review: Current Research Issues on Crypto-currency and its Application in Financial Sectors
}

\author{
Lim Hong $\operatorname{Hin}^{1}$ \\ ${ }^{1}$ Liberty University, USA \\ Correspondence: Lim Hong Hin, Liberty University, USA. E-mail: hlim10@liberty.edu
}

Received: December 16, 2019

Accepted: January 20, 2020

Online Published: February 27, 2020

doi:10.5539/ijbm.v15n3p145

URL: https://doi.org/10.5539/ijbm.v15n3p145

\begin{abstract}
This research article is based on the current research issues on crypto-currency and its application in financial sectors. The researcher stated that crypto- currency attracted a significant attention and has been adopted in numerous applications, such as smart grid and Internet of Things (IoT) have a high scalability barrier for block-chain which limits its ability to support services with frequent transactions. In this research paper the researcher emphasized that some of the significant research issues on decentralized, peer to peer financial networks face risks such as fraud and suspicious trading, volatile price fluctuations, regularity uncertainty, and instability in financial sectors. This research study shows the huge growth of online users, virtual word concept which are created a new business phenomenon to facilitate financial activities such as buying, selling and trading. This research article analyzed the user's expectation of future of crypto-currency in financial sectors at different significant level. In addition to this the researcher also discussed a statistical report which is adopted from the Statistic to provide a future direction of money regulatory in financial sector.
\end{abstract}

Keywords: crypto-currency, bit coin, block-chain

\section{Introduction}

Crypto- currency is am internet-based medium for exchanging transaction valuation in financial sectors which cryptographically functions to conduct financial transactions. Crypt currency leverages block chain technology to gain decentralized, transparency, and immutability. In this research article the research emphasized that some of the facts about crypto-currency which are stated as:

1. Crypto-currency is not controlled by any central authority. The decentralized nature of the block chain makes crypto-currency theoretical immune to the old age of government and interferences.

2. Crypto-currency is an alternative approach of exchanging the money valuation between transactions in financial sectors.

3. Crypto-currency can be sent directly between parties in financial sectors, the transaction can be done with minimal processing fees which are allowed to avoid the steep fees charged by the traditional financial sectors.

4. The crypto-currency have become a global phenomenon known to most people who need to know about crypto currency and the sheer that they can bring into global economy system.

\subsection{What Is Crypto-Currency?}

In this research article the researcher emphasized crypto-currency is one the digital payment systems which have a limited entries in a database and can exchange without fulfilling specific conditions. In current scenario the money is all about a verified entry in some kinds of account, balance, and transactions. The crypto-currency is defined as internet based medium of exchanging which uses crypto-graphically functions to conduct financial transaction in real world. The crypto-currency leverage block chain technology to gain decentralization, transparency and immutability. The researcher focused on that mechanism ruling the database of crypto-currency with respect to bit coin consisted of networks during transactions way. In crypto-currency every peer has a record of complete history of all transactions and thus for balance of every accounts [5]. In crypto-currency, a transaction is a file that it is encrypted with public and private keys, after signed a transaction is broadcast in the networks sent from one peer to every other peer in the network on the basis of P2P technology. 
Yli-Huumo et al. (2016) stated that block chain is a decentralized technology and developed first for bit con-crypto-currency for storing and managing the business transaction in smooth and secure way. In this research paper the researcher conducted a systematic mapping study with the goal of collecting all relevant research on block chain technology. The majority of the research is focusing on relieving and improving limitations of block chain from privacy and security concerns.

\section{Related Works}

Corina Sas and Irni Eliana Khairuddin (2015) emphasized that bit coin is a crypto-currency is a digital money which are completely differs in several ways of traditional us of money. It does not require digital wallets IDs. In this research paper the researcher stated that crypto-currency is the model of trust which is proposed to explore trust challenges raised by the bit coin technology. Iuon-Chang Lin and Tzu-Chun Liao (2017) emphasized that block chain technology is one of the most popular issues in recent years which reflects to great influence on business transactions. The block chain technology may bring more reliable and convenient services, the security issues and challenging behind this innovative technique in financial sectors.

Mohamed Amine Ferrag et al. (2019) stated that block chain technology protocols for the Internet of Things (IoT) is playing one of the significant role in financial sectors to provide smooth transactions. The researcher focused on the basic patterns of Internet of Things (IoT) which are associated to Inter of Energy, Internet of Clouds, Edge computing and others areas which are significant for the security point of view for financial transactions in business Industries. The Internet of Things (IoT) networks have a different categories namely identified based attacks, manipulations based attacks, and service based attacks. Adrian et al. (2015) emphasized that crypto-currency has been regarded as a phenomenon and extended to rapid growth by huge swings to control the business transactions in financial sectors.

Fang Dai et al. (2018) stated that crypto-currency is a digital payments which is based on decentralized distributed database system for data management solutions. The researchers also emphasized that this is one of the most trustable technology which are providing security, anonymity and data integrity without the need of any third party. Kimchai Yeow et al. (2019) emphasized that Internet of Things (IoT) is geared towards number of devices edge centric computing to offers high bandwidth, low latency, and improved connectivity.

Vovchenko et al. (2017) emphasized that crypto-currency is one of the category of virtual currencies which given to development of risk and threats to national security and money laundering cases, criminal money and terrorist funding. The researcher suggested that with minimal negative impact of crypto-currency adopted for management centre in virtual currency infrastructure. Chinmay A. Vyas, Munindra Lunagaria (2014) focused on the unique characteristics of Bit-coin as a crypto-currency and major security issues regarding the mining process and transaction process of bit coin. The researcher emphasized that security is one of the major concern for all transactions for exchanging money to control their business operations in financial sectors.

Bela Gipp et al. (2015) discussed on process of improving the crypto-currency at given point in time for trusting web based services that uses the decentralized distributed database. The block chain technology chain to store anonymous, tamper proofs timestamp for digital consent. Ruizhe Yang et al. (2019) stated block chain as the underlying technology of crypto-currency which is having a significant impact on business transactions such as smart grid, Internet of Things. The scalability of block chain technology enhancement, self -organizations, integrations, resource management and wide spread development.

Kirillova, et al. (2018) examined the legal nature of bit coin, life coin, web money, ripple and virtual currencies. The researcher stated that licensing of bit coin in digital currency is playing a significant role in financial sectors. This research study is conducted on mining crypto- trading at international levels to prevent the abuse of virtual currencies for money laundering and terrorism financing. Karl Sigler (2018) emphasized that crypto-currency is an esoteric experiment to one of the hottest topics in finance and technology field. Crypto-currency is jacking for target machine which is infected with mining malware.

A. Yu. Simanovskiy (2018) emphasized that the economic nature of crypto-currency, risks that arise from its use of electronic turn over servicing and consequences of different opinions for portable legalizations. The major way of crypto- currency existences is Ponzi scheme for limiting negative economic and social cost of cryptocurrency use. Irwin and Milad (2016) emphasized that crypto-currency, more specifically on terror funding, new way of payment technology and value transfer system. The researcher also emphasized that the present role of crypto-currency for transfer system to facilitate the funding, planning, seamless and its significant implementation to protect terror attacks.

Malhotra, Yogesh (2013) stated that technical focus cryptographic proof of work in the context of virtual crypto- 
currencies which is based on natural stage in the evolution of global finance to ensure its integrity for reliable medium exchange in financial sectors. Danny Bradbury (2013) worked on extensively on the project to open source community subject to attacks on numerous occasions, and is in danger zone in financial sectors to minimize interferences for criminal to have found a way to subvert.

Adam Abdullah and Rizal Mohr Nor(2018) provided a conceptual framework of crypto-currency for purchasing power of money and to measure the comparative performance of price stability, which retains in store of value in the terms of monetary performance and price stability in financial sectors. Casinoa Thomas et al. (2019) provided a systematic review of block chain technology and its application to highlights on disruptive technology of streaming continuous expanding block chain technology.

Remy Remigius Zgraggen (2019) focused on the crypto-currency based insurance contact for the relevant legal frameworks for public and private laws. Jamal Bouoiyour, Refk Selmi and Aviral Kumar Tiwari (2015) stated that crypto-currency is having one of the significant approach financial sectors to control secure and reliable transactions.

\section{Problem Statement and Research Issues}

The researcher emphasized that scalability is one of the significant barrier in block chain technology for exchanging transaction and money value for their business. Security is also one of the major concern in decentralized data storage of the networks. The researcher stated that some of the current research issues and its significant impact in financial sectors. For making more reliable and enhance computing services over internet, the encryption is providing a secure transaction in financial sectors.

In terms of secure and reliable transactions, the crypto-currency is one of the significant components for exchanging money value and their significant approach for smooth transactions. The researcher stated that some of the significant research issues on crypto-currency and it application in financial sectors.

1. To study the various security factors of crypto-currencies in financial sectors.

2. To study the current research issues on crypto-currencies and its significant application in financial sectors.

\section{Research Design and Methodology}

This research paper is designed to identify the current research issues on crypto-currency and its significant application in financial sectors. The researcher developed a proposed research model to emphasize that the security barriers in crypto- currency due to decentralized its data structure, a block of data is travelling over the internet during data transactions in financial sectors. The research collected data on current research issues from research portals, books, magazines, and other sources of data collections and developed a critical review on current research issues and its significant approach in financial sectors. During this research study the research found some of significant research issues on crypto-currency. The researcher designed this research article by the shortage of comprehensive reviews on decentralized consensus system for edge centric Internet of Things (IoT) which controls such as data structure, scalable consensus systems models such as vulnerable wallets, when it comes into hacking attacks and theft, hackers and cyber-attacks.

\section{Statistical Report on Crypto Currency}

In this research article, the researcher presented a statistical report analysis in between 2013 to 2018 of market capitalization of crypto-currencies in U.S. Dollars.

The researcher found the different statistics on crypto-currency with respect to security challenges and its significant usage in financial sectors.

Table 1. Market capitalization of crypto-currencies from 2013 to 2018 (in billion U.S. dollars)

\begin{tabular}{cc}
\hline Year & Market capitalization of crypto-currencies from 2013 to 2018 (in billion U.S. dollars) \\
\hline 2013 & 10.62 \\
2014 & 5.54 \\
2015 & 7.09 \\
2016 & 17.7 \\
2017 & 566.26 \\
2018 & 128.78 \\
\hline
\end{tabular}

Source. Statista-Market Capitalization of Crypto-currencies from 2013 to 2018 (In Billion U.S. Dollars) 


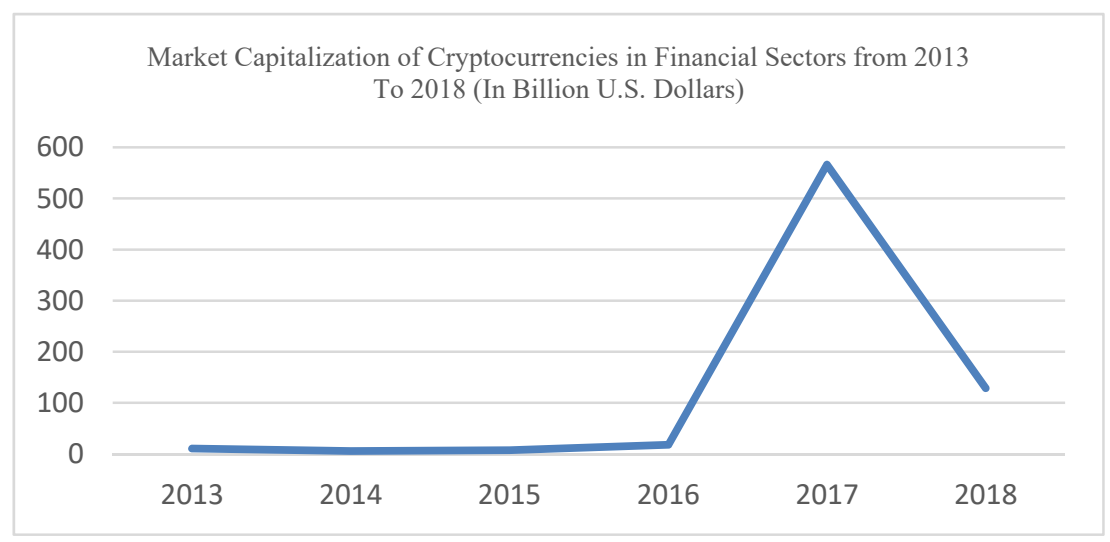

Figure 1. Analysis of market capitalization of crypto-currencies from 2013 to 2018 (in billion U.S. dollars)

The statistic presents the market capitalization of crypto currencies from 2013 to 2018 . The value of crypto currencies globally in 2018 amounted to 128.78 billion U.S. dollars. A crypto currency is a digital currency that is created and managed through the use of advanced encryption techniques known as cryptography. Crypto currency made the leap from being an academic concept to (virtual) reality with the creation of Bitcoin in 2009 . While Bitcoin attracted a growing following in subsequent years, it captured significant investor and media attention in April 2013 when it peaked at a record \$266 per bitcoin after surging 10-fold in the preceding two months. Bitcoin sported a market value of over $\$ 2$ billion at its peak, but a $50 \%$ plunge shortly thereafter sparked a raging debate about the future of crypto currencies in general and Bitcoin in particular. So, will these alternative currencies eventually supplant conventional currencies and become as ubiquitous as dollars and euros someday? Or are crypto currencies a passing fad that will flame out before long? The answer lies with Bitcoin.

\section{Research Issues on Crypto Currency}

In this research article, the researcher emphasized that crypto currencies has a decades-long pedigree in academia, but decentralized crypto currencies (starting with Bitcoin in 2009) have taken the world by storm. Aside from being a payment mechanism "Native to the Internet," the underlying block chain technology is touted as a way to store and transact everything from property records to certificates for art and jewelry. However, Researchers have embraced crypto currencies with gusto and have contributed important insights.

1. Anonymity, Privacy, and Confidentiality: Anonymity in crypto currencies is a matter of not just personal privacy, but also confidentiality for enterprises. Each transaction is accompanied by a cryptographic, publicly verifiable proof of its own validity. Roughly, the proof ensures that the amount being spent is no more than the amount available to spend from that address.

2. Endpoint Security:Turning to security, the Achilles' heel of crypto currencies has been the security of endpoints, or the devices that store the private keys that control one's coins

3. Smart Contracts: One of the hottest areas within crypto currencies, so-called smart contracts are agreements between two or more parties that can be automatically enforced without the need for an intermediary.

4. Overcoming the Pitfalls: Crypto currencies implement many important ideas: digital payments with no central authority, immutable global ledgers, and long-running programs that have a form of agency and wield money. As researcher seen, there are pitfalls for the unwary in using and applying crypto currencies: privacy, security, and interfacing with the real world.

5. Deep Learning: DNNs (deep neural networks) have evolved to a state-of-the-art technique for machine-learning tasks ranging from computer vision to speech recognition to natural language processing. Deep-learning algorithms, however, are both computationally and memory intensive, making them power-hungry to deploy on embedded systems. Running deep-learning algorithms in real-time at sub watt power consumption would be ideal in embedded devices, but general-purpose hardware is not providing satisfying energy efficiency to deploy such a DNN.

6. Optimized Data Flow: Deep-learning algorithms are memory intensive, and accessing memory consumes energy more than two orders of magnitude more than ALU (arithmetic logic unit) operations. This is realized by exploiting local data reuse of filter weights and feature map pixels (i.e., activations) in the high-dimensional convolutions, and by minimizing data movement of partial sum accumulations. 


\section{Application of Crypto-Currency in Financial Sectors}

The researcher stated that some of the significant usage of crypto-currencies in financial sector which is defined as crypto currency is a digital asset designed to work as a medium of exchange that uses strong cryptography to secure financial transactions, control the creation of additional units, and verify the transfer of assets that uses cryptography for security" making it difficult to counterfeit. Since it is not issued by a central authority, governments.

1. Fraud-Proof: When crypto currency is created, all confirmed transactions are stored in a public ledger. All identities of coin owners are encrypted to ensure the legitimacy of record keeping. Because the currency is decentralized, you own it. Neither government nor bank has any control over it.

2. Identity Theft: The ledger ensures that all transactions between "digital wallets" can calculate an accurate balance. All transactions are checked to make sure.

3. Instant Settlement: Block chain is the reason why crypto currency has any value. Ease of use is the reason why crypto currency is in high demand. All you need is a smart device, an internet connection and instantly you become your own bank making payments and money transfers.

4. Accessible: There are over two billion people with access to the Internet who don't have rights to use to traditional exchange systems.

\section{The Future Dimensions of Crypto Currency}

The researcher emphasized that the future of crypto-currency in financial sectors and its significant usage which will be harder to surmount is the basic paradox that bedevils crypto currencies which require more regulation and government scrutiny are likely to attract, which erodes the fundamental premise for their existence. The researcher analyzed that some economic analysts predict a big change in crypto is forthcoming as institutional money enters the market. Moreover, there is the possibility that crypto will be floated on the NASDAQ, which would further add credibility to block chain and its uses as an alternative to conventional currencies. Some predict that all that crypto needs are a verified exchange traded fund (ETF). An ETF would definitely make it easier for people to invest in Bit coin, but there still needs to be the demand to want to invest in crypto, which some say may not automatically be generated with a fund.

\section{Conclusion}

Finally, the researcher concluded that crypto-currency is playing one of the significant roles for business transaction for smooth transactions in financial sectors. In this research article the researcher stated that to avoid fraud and hackers attacks with respect to safe guard and protection. Decentralization is also one the security concern data management in financial sectors without being tax evasion, money laundering and other activities. In currency scenario the most popular crypto- currency that fall in between heavily regulated and fiat currencies which decided to intervene and warn that bit coin could be the next enable bubble. The government prohibited the trading of crypto-currency due to lack of control and market unpredictability. The centralization of hashing power in the hands of a few or in particular geographic areas which are facing main challenges

\section{References}

Adam, A., \& Rizal, M. (2018). A Framework for the Development of a National Crypto-Currency. International Journal of Economics and Finance, 10(9).

Adrian, W. K., Cheung, E. R., \& Su, J. J. (2015). Crypto-currency bubbles: a usage of the Phillips-Shi-Yu (2013) technique on Mt. Gox bit coin costs. Journal Applied Economics, 47(23), 2348-2358. https://doi.org/10.1080/00036846.2015.1005827

Bela, G., Norman, M., \& André, G. (2015). Decentralized Trusted Time stamping using the Crypto Currency Bitcoin, Cryptography and Security. Newport Beach, CA, USA.

Casinoa, T. K., Dasaklisb, C. P. (2019). A exact composing review of block chain-based applications: Current status, gathering and open issues. Telematics and Informatics, 36, 55-81. https://doi.org/10.1016/j.tele.2018.11.006.

Chinmay, A., Vyas, M. L. (2014). Security Concerns and Issues for Bitcoin, International Journal of Computer Applications ${ }^{\circledR}$ (IJCA) (0975-8887). National Conference cum Workshop on Bioinformatics and Computational Biology, NCWBCB-2014.

Corina, S., \& Irni, E. K. (2015). Exploring Trust in Bit coin Technology: A Framework for HCI Research, Oz CHI '15 Proceedings of the Annual Meeting of the Australian Special Interest Group for Computer Human 
Interaction, ACM New York, NY, USA. https://doi.org/10.1145/2838739.2838821

Dai, F. F., Yue, S., Nan, M., Liang, W., \& Ye, Z. G. (2018). From Bitcoin to digital security: A close to examination of square chain application and security issues, 2017 fourth International Conference on Systems and Informatics (ICSAI), IEEE Xplore. https://doi.org/10.1109/ICSAI.2017.8248427

Danny, B. (2013). The issue with Bitcoin. Computer Fraud and Security, (11), 5-8. https://doi.org/10.1016/S1361-3723(13)70101-5.

Irwin, A., \& Milad, G. (2016). The use of crypto-fiscal structures in sponsoring severe jihad. Journal of Money Laundering Control, 19(4), 407-425. https://doi.org/10.1108/JMLC-01-2016-0003

Jamal, B., Refk, S., \& Aviral, K. T. (2015). Is Bitcoin Business Income Or Speculative Foolery? New Ideas through an Improved Frequency Domain Analysis, World Scientifics. Annals of Financial Economics, 10(1). https://doi.org/10.1142/S2010495215500025

Karl, S. (2018). Crypto-jacking: how advanced guilty parties are abusing the crypto-currency impact. Computer Fraud and Security, 9, 12-14. https://doi.org/10.1016/S1361-3723(18)30086-1

Kimchai, Y., Abdullah, G., Raja, W. A., Joel, J. P. C., Rodrigues, K. K. O. (2019). Decentralized Consensus for Edge-Centric Internet of Things: A Review, Taxonomy, and Research Issues, Internet-of-Things (IoT) Big Data. Trust Management, 6, 1513-1524. https://doi.org/10.1109/ACCESS.2017.2779263

Kirill, O., Elena, A., Pavlyuk, A. V., Mikhaylova, I. A. Zulfugarzade, T. E. Z. (2018). Bitcoin, Lifecoin, Name coin: The Legal Nature of Virtual Currency, Sergey. Journal of Advanced Research in Law and Economics, 9(1), 119-126. https://doi.org/10.14505/jarle.v9.1(31).16

Lin, I. C., \& Liao, T. C. (2017). A Survey of Block chain Security Issues and Challenges, International Journal of Network Security, 19(5), 653-659. https://doi.org/10.6633/IJNS.201709.19 (5).01) 653.

Malhotra, Y. (2013). Bitcoin Protocol: Model of 'Cryptographic Proof' Based Global Crypto-Currency and Electronic Payments System. http://dx.doi.org/10.2139/ssrn.2911623

Mohamed, A. F., Makhlouf, D., Mithun, M., Abdelouahid, D., \& Leandros, M. (2019). Square chain Technologies for the Internet of Things: Research Issues and Challenges. IEEE Internet of Things Journal, 6(2), 2188-2204. https://doi.org/10.1109/JIOT.2018.2882794

Remy, R. Z. (2019). Sharp Insurance Contracts Based on Virtual Currency: Legal Sources and Chosen Issues, IECC '19 Proceedings of the 2019. International Electronics Communication Conference, 99-102, https://doi.org/10.1145/3343147.3343161

Vovchenko, N. G., Tishchenko, E. N., Epifanova, T. V., \& Gontmacher, M. B. (2017). Electronic currency: the potential perils to national security and methods to restrain them. European Research Studies Journal, 20(1), $36-48$.

Yang, R. Z., Yu, F. R., Si, P. B., Yang, Z. X., \& Zhang, Y. H. (2019). Integrated Block chain and Edge Computing Systems: A Survey, Some Research Issues and Challenges. IEEE Communications Surveys and Tutorials, 21(2), 1508-1532. https://doi.org/10.1109/COMST.2019.2894727

Yli-Huumo, J., Ko, D., Choi, S., Park, S., \& Smolander, K. (2016). Where Is Current Research on Block chain Technology? — A Systematic Review. PLoS ONE, 11(10). https://doi.org/10.1371/journal.pone.0163477

Yu, A. S. (2018). On the issue of crypto-currency money related nature, Prosy Economiki, N. P. Redaktsiya zhurnala. Voprosy Economiki.

\section{Copyrights}

Copyright for this article is retained by the author(s), with first publication rights granted to the journal.

This is an open-access article distributed under the terms and conditions of the Creative Commons Attribution license (http://creativecommons.org/licenses/by/4.0/). 